\title{
AN ASPECT OF THE INVARIANT OF DEGREE 4 OF THE BINARY QUINTIC
}

\author{
by W. L. EDGE
}

(Received 31st May 1985)

\section{Introduction}

1. A binary form of odd degree,

$$
f(x, y) \equiv \sum_{r=0}^{2 m+1}\left(\begin{array}{c}
2 m+1 \\
r
\end{array}\right) a_{r} x^{2 m+1-r} y^{r} \equiv a_{x}^{2 m+1} \equiv b_{x}^{2 m+1},
$$

has a quadratic covariant $\Gamma,(a b)^{2 m} a_{x} b_{x}$ in Aronhold's notation, and the discriminant $\Delta$ of $\Gamma$ is an invariant of $f$. For $m=2 \Delta$ was obtained by Cayley in 1856 [3, p. 274]; it was curiosity as to how $\Delta$ could be interpreted geometrically that triggered the writing of this note. An interpretation, in projective space $[2 m+1]$, that does not seem to be on record, of $\Gamma$ and $\Delta$ is found below. If $m=1$ one has merely the Hessian and discriminant of a binary cubic whose interpretations in the geometry of the twisted cubic are widely known [5, pp. 241-2].

There is a standard mapping

$$
f(x, y) \equiv \sum_{r=0}^{n}\left(\begin{array}{l}
n \\
r
\end{array}\right) a_{r} x^{n-r} y^{r} \rightarrow\left(a_{0}, a_{1}, \ldots, a_{n}\right)
$$

of a binary $n-i c$ onto a point $P$ in a projective space $[n]$ wherein $\left(x_{0}, x_{1}, \ldots, x_{n}\right)$ are homogeneous coordinates. The focus, so to call it, of this map is the rational normal curve $C$ in Clifford's canonical form

$$
x_{i}=(-t)^{i} ; \quad i=0,1, \ldots, n
$$

its points map those $f$ that are perfect $n$th powers. For $n=4$ the geometry of the rational normal quartic was used by Brusotti [2] to interpret the concomitants of a binary quartic, but only odd integers $n=2 m+1$ concern us here. For $m=2$ a note [8] by Todd is relevant but his account does not involve, as ours will, the manifolds generated by the spaces osculating $C$.

These osculating spaces dominate a paper [1] by Baker who shows that the coefficients in the polarised form $(a b)^{2 r} a_{x}^{n-2 r} b_{y}^{n-2 r}$ provide all the quadrics containing all the osculating $[r-1]$ of $C$. Our concern is restricted here to $n=2 m+1, r=m$; this 
produces a net $N$ of quadrics. But we identify the singular quadrics of $N$, their envelope being our main objective.

\section{The rational normal quintic and the net of quadrics containing all its tangents}

2. Suppose therefore, until after Section 6 , that $n=5$ in (1.1). The chordal [4] spanned by the five points $t=\alpha_{0}, \alpha_{1}, \alpha_{2}, \alpha_{3}, \alpha_{4}$ of $C$ is

$$
e_{5} x_{0}+e_{4} x_{1}+e_{3} x_{2}+e_{2} x_{3}+e_{1} x_{4}+x_{5}=0
$$

where

$$
\theta^{5}-e_{1} \theta^{4}+e_{2} \theta^{3}-e_{3} \theta^{2}+e_{4} \theta-e_{5} \equiv \prod_{j=0}^{4}\left(\theta-\alpha_{j}\right)
$$

and this is so whatever confluences may occur among the $\alpha_{j}$. In particular $\omega_{4}(\alpha)$, the osculating [4] at $t=\alpha$, is

$$
\alpha^{5} x_{0}+5 \alpha^{4} x_{1}+10 \alpha^{3} x_{2}+10 \alpha^{2} x_{3}+5 \alpha x_{4}+x_{5}=0
$$

so that the zeros $x / y$ of $f$ are the parameters of the contacts of those $\omega_{4}$ that contain $P$. Osculating spaces of lower dimension are identified by simultaneous linear equations; for example, three equations identifying $\omega_{2}(\alpha)$ are

$$
\alpha^{3} x_{k}+3 \alpha^{2} x_{k+1}+3 \alpha x_{k+2}+x_{k+3}=0, \quad k=0,1,2
$$

The basic geometry of $C$ is described in [6], its ranks being given on p. 95. The facts are that

the tangents $\omega_{1}$ generate a scroll $\Omega_{2}^{8}$,

the osculating planes $\omega_{2}$ generate a threefold $\Omega_{3}^{9}$,

the osculating solids $\omega_{3}$ generate a primal $\Omega_{4}^{8}$.

It is sometimes convenient to homogenise (1.1) as $x_{i}=(-1)^{i} u^{i} v^{5-i}$; then partial differentiations produce points spanning $\omega_{1}, \omega_{2}$, and so on. In particular $\omega_{1}(t)$ is spanned by

and

$$
\text { a: } \quad 0, \quad-1, \quad 2 t, \quad-3 t^{2}, \quad 4 t^{3}, \quad-5 t^{4}
$$

$$
b: \quad 5,-4 t, \quad 3 t^{2},-2 t^{3}, t^{4}, \quad 0 \text {. }
$$

3. Consider now the possibility of $\Omega_{2}^{8}$ lying on a quadric $Q: \Sigma a_{r s} x_{r} x_{s}=0$; the matrix $\left(a_{r s}\right)$ is symmetric, $a_{r s}=a_{s r}$, both $r$ and $s$ running from 0 to 5 ; products $x_{r} x_{s}(r \neq s)$ each occur twice, squares only once. If $\omega_{1}(t)$ is on $Q$ then $Q$ contains both $a$ and $b$ conditions $I_{1}$ and $I_{2}$ of incidence-while $a, b$ are also conjugate-condition $J$. All three conditions are linear in the $a_{r s}$; each demands that an octavic polynomial in $t$ is zero, 
while if all $\omega_{1}$ are on $Q$ these polynomials are to be zero identically, whatever $t$. But in any of $I_{1}, I_{2}, J$ those $a_{r s}$ with the same $r+s$ multiply the same power of $t$, this power being $r+s-2$ in $I_{1}$ (wherein no $a_{0 s}$ occurs), $r+s$ in $I_{2}$ and $r+s-1$ in $J$ (wherein $a_{00}$ does not occur); so parallels to the secondary diagonal of $\left(a_{r s}\right)$ can be handled independently. A glance at the information provided by the lower and higher values of $r+s$ will show that all $a_{r s}$ are zero for which $r+s$ is any of $0,1,2,3 ; 7,8,9,10$ so that the only non-zero $a_{r s}$ are those in the secondary diagonal $(r+s=5)$ itself and the two contiguous parallels $(r+s=4,6)$.

The three conditions are

$$
\begin{gathered}
I_{1}: 0=a_{11}-4 a_{12} t+\left(4 a_{22}+6 a_{13}\right) t^{2}-\left(8 a_{14}+12 a_{23}\right) t^{3}+\cdots \\
J: 0=-5 a_{01}+\left(4 a_{11}+10 a_{02}\right) t-\left(11 a_{12}+15 a_{03}\right) t^{2}+\left(20 a_{04}+14 a_{13}+6 a_{22}\right) t^{3}+\cdots \\
I_{2}: 0=25 a_{00}-40 a_{01} t+\left(16 a_{11}+30 a_{02}\right) t^{2}-\left(20 a_{03}+24 a_{12}\right) t^{3}+\left(10 a_{04}+16 a_{13}+9 a_{22}\right) t^{4}+\cdots
\end{gathered}
$$

giving in succession

$$
a_{11}=a_{01}=a_{00}=a_{12}=a_{02}=a_{03}=0
$$

while working down from $t^{8}$ as has been here worked upwards from the constant terms one would find

$$
a_{44}=a_{54}=a_{55}=a_{43}=a_{53}=a_{52}=0 \text {. }
$$

When $r+s=4$ the three conditions are

$$
6 a_{13}+4 a_{22}=20 a_{04}+14 a_{13}+6 a_{22}=10 a_{04}+16 a_{13}+9 a_{22}=0
$$

three linearly dependent constraints upon the $a_{r 3}$ which hold when $a_{04}:-a_{13}: a_{22}$ are in the ratios 1:4:6 of the binomial coefficients and so affording a quadric $x_{0} x_{4}-4 x_{1} x_{3}+$ $3 x_{2}^{2}=0$ featuring a familiar trinomial. As $C$ is invariant under the involutory permutation $\left(x_{0} x_{5}\right)\left(x_{1} x_{4}\right)\left(x_{2} x_{3}\right)$ imposed by $t \leftrightarrow t^{-1}$, i.e. by harmonic inversion in the two planes

$$
x_{0}=x_{5}, x_{1}=x_{4}, x_{2}=x_{3} \text { and } x_{0}=-x_{5}, x_{1}=-x_{4}, x_{2}=-x_{3}
$$

$\Omega_{2}^{8}$ also lies on the quadric $x_{5} x_{1}-4 x_{4} x_{2}+3 x_{3}^{2}=0$ as could also have been found by imposing $I_{1}, I_{2}, J$. As for $r+s=5$ the conditions require

$$
4 a_{14}+6 a_{23}=25 a_{05}+17 a_{14}+13 a_{23}=4 a_{14}+6 a_{23}=0
$$

of which the first and third both give $a_{14}: a_{23}=-3: 2$, a consistency explained by the 
invariance under $\left(x_{0} x_{5}\right)\left(x_{1} x_{4}\right)\left(x_{2} x_{3}\right) . J$ then demands

$$
a_{05}: a_{14}: a_{23}=1:-3: 2
$$

so that $x_{0} x_{5}-3 x_{1} x_{4}+2 x_{2} x_{3}=0$ also contains $\Omega_{2}^{8}$. The outcome is that (cf. [1], pp. 137 and 143)

$$
\underline{\Omega_{2}^{8} \text { is the base surface of a net } N \text { of quadrics } Q,}
$$

say

$$
p\left(x_{0} x_{4}-4 x_{1} x_{3}+3 x_{2}^{2}\right)-q\left(x_{0} x_{5}-3 x_{1} x_{4}+2 x_{2} x_{3}\right)+r\left(x_{1} x_{5}-4 x_{2} x_{4}+3 x_{3}^{2}\right)=0 .
$$

Readers acquainted with texts on invariants will have remarked that, on replacing $x_{i}$ by $a_{i}$, the three quadrics on which we have based $N$ become the coefficients in $\Gamma([3], \mathrm{p}$. 273; [7],p. 206). But the geometry has more to say.

\section{The cones of the net and the interpretation of their envelope}

4. The symmetric matrix of a quadric of $N$ is

$$
(N)=\left[\begin{array}{cccccc}
. & . & . & . & p & -q \\
\cdot & . & . & -4 p & 3 q & r \\
\cdot & . & 6 p & -2 q & -4 r & . \\
\cdot & -4 p & -2 q & 6 r & . & . \\
p & 3 q & -4 r & . & . & . \\
-q & r & . & . & . & .
\end{array}\right] .
$$

The Laplace expansion on the top three and bottom three rows, in which only two nonzero products occur, or a triple Laplace expansion on the two top, two middle and two bottom rows, in which only four non-zero triple products occur, gives

$$
|(N)|=36\left(r p-q^{2}\right)^{3}
$$

so that the only cones in $N$ have $q^{2}=r p$ or, say,

$$
p: q: r=\rho^{2}:-\rho: 1 .
$$

When this substitution is made in $(N)$ only a single linear dependence between the rows emerges, namely

$$
R_{1}-\rho R_{2}+\rho^{2} R_{3}-\rho^{3} R_{4}+\rho^{4} R_{5}-\rho^{5} R_{6} \equiv 0
$$

so that the resulting matrix has rank 5 and the cone has a single point for vertex, indeed 
the point on $C$ with $t=\rho$. The cones of $N$ thus compose a family of index 2 and their envelope, the locus of points $P$ such that the two cones of $N$ passing through $P$ coincide, is the quartic primal $K$ with equation

$$
\left(x_{0} x_{5}-3 x_{1} x_{4}+2 x_{2} x_{3}\right)^{2}=4\left(x_{0} x_{4}-4 x_{1} x_{3}+3 x_{2}^{2}\right)\left(x_{1} x_{5}-4 x_{2} x_{4}+3 x_{3}^{2}\right)
$$

$\Omega_{2}^{8}$ is a double surface on $K$.

This is the sought interpretation. The situation may be described as follows.

There is a net $N$ of quadrics containing all the tangents of a rational normal quintic $C$; the singular members of $N$ are all point-cones with vertices on $C$. Through any point $P$ pass two of these cones, their vertices having for parameters on $C$ the two zeros of a quadratic covariant of the binary quintic $f$ mapped by $P$. If $P$ is such that these two cones are coincident it lies on the quartic primal $K$ and maps an $f$ for which the invariant of degree 4 is zero.

Each cone of $N$ touches $K$ along a quartic threefold containing $\Omega_{2}^{8}$. In the geometry of the non-singular plane quartic the contacts of any two contact conics of the same system are eight points on a conic, and the analogous circumstance holds for $K$; any non-singular quadric of $N$ meets $K$ in a pair of the quartic threefolds. These are the contacts of those cones for which $\rho$ satisfies $p-2 q \rho+r \rho^{2}=0$.

Now that the equation of $K$ has been found it is apparent that $K$ contains $\Omega_{3}^{9}$, i.e. every osculating plane $\omega_{2}$. For it manifestly contains $\omega_{2}(0)$, whose equations (cf. 2.2) are $x_{3}=x_{4}=x_{5}=0$, while it has been defined geometrically in reference to the whole of $C$ with no restriction to any coordinate system.

5. Since a rational plane quintic has six nodes or their equivalent a plane of general position in [5] meets six chords of $C$ : the chords of $C$ generate a sextic threefold $M_{3}^{6}$. The surface common to $M_{3}^{6}$ and a quadric of $N$ includes $\Omega_{2}^{8}$; the residue is a quartic scroll. For any chord of $C$ is, as $\lambda, \mu$ vary, traced by the point $x_{i}=(-1)^{i}\left(\lambda \phi^{i}+\mu \psi^{i}\right)$; the result of substituting these $x_{i}$ in (3.1) is

$$
\lambda \mu\{p+q(\phi+\psi)+r \phi \psi\}(\phi-\psi)^{4}=0
$$

so that those chords which, in addition to all tangents, lie on (3.1) are joins of the pairs of the involution

$$
p+q(\phi+\psi)+r \phi \psi=0
$$

Such joins are known ([6], p. 97) to generate a rational normal quartic scroll. But the involution can degenerate, the scroll becoming the quartic cone of chords through a point of $C$; this occurs whenever $q^{2}=r p, p: q: r=\rho^{2}:-\rho: 1$, the "involution" consisting of all chords through $t=\rho$.

As $C$, on the double surface of $K$, is at least a double curve any chord of $C$ that meets $K$ at a point not on $C$ lies wholly on $K$; the surface, of order 24 , common to $K$ and $M_{3}^{6}$ 
is a scroll. As $\Omega_{2}^{8}$ is on $M_{3}^{6}$ and is double on $K$ it it counts twice in the intersection, leaving an octavic residue; this turns out to be $\Omega_{2}^{8}$ again. For when $x_{i}$ is replaced by $(-1)^{i}\left(\lambda \phi^{i}+\mu \psi^{i}\right)$ in (4.1) the outcome is $\left\{\lambda \mu(\phi+\psi)(\phi-\psi)^{4}\right\}^{2}=4\left\{\lambda \mu(\phi-\psi)^{4}\right\}\left\{\lambda \mu \phi \psi(\phi-\psi)^{4}\right\}$

$$
\lambda^{2} \mu^{2}(\phi-\psi)^{10}=0
$$

so that no chord of $C$ lies entirely on $K$ unless it is a tangent: $K$ meets $M_{3}^{6}$ in $\Omega_{2}^{8}$ reckoned thrice.

The net of quadrics and the osculating planes

6. The developable surface $\Omega_{2}^{8}$ has the same tangent plane $\omega_{2}(t)$ at every point of $\omega_{1}(t)$. Since the tangent prime at any point of $\omega_{1}(t)$ to any quadric $Q$ of $N$ contains the tangent plane of $\Omega_{2}^{8}$ there $\omega_{2}(t)$ is in the polar solid of $\omega_{1}(t)$ with respect to $Q$. But this solid meets $Q$ in a pair of planes through $\omega_{1}(t)$ so that $\omega_{2}(t)$, unless it lies on $Q$, meets $Q$ only in $\omega_{1}(t)$ counted twice. It can be proved by correspondence theory that two osculating planes of $C$ lie wholly on $Q$ and these, in the present context, can be identified by elementary algebra. For the three equations (2.2) are equivalent to asserting that, for $s=0,1,2,3$ the four fractions $\left(\alpha^{2} x_{s}+2 \alpha x_{s+1}+x_{s+2}\right) /(-\alpha)^{s}$ are equal and so, again by (2.2), the points of $\omega_{2}(\alpha)$ satisfy the three quadratic conditions

$$
\sum_{r=0}^{3}(-1)^{r}\left(\begin{array}{l}
3 \\
r
\end{array}\right)\left(\alpha^{2} x_{3-r}+2 \alpha x_{4-r}+x_{5-r}\right) x_{k+r}=0, \quad k=0,1,2 .
$$

If $k=0$ the terms in $\alpha^{2}$ cancel one another, as do those in $\alpha$ when $k=1$ and those without $\alpha$ when $k=2$. The full equation when $k=2$ is

$$
\alpha^{2}\left(x_{3} x_{2}-3 x_{2} x_{3}+3 x_{1} x_{4}-x_{0} x_{5}\right)+2 \alpha\left(x_{4} x_{2}-3 x_{3}^{2}+3 x_{2} x_{4}-x_{1} x_{5}\right)=0
$$

i.e.

$$
\alpha^{2}\left(x_{0} x_{5}-3 x_{1} x_{4}+2 x_{2} x_{3}\right)+2 \alpha\left(x_{1} x_{5}-4 x_{2} x_{4}+3 x_{3}^{2}\right)=0
$$

with the quadrics of $N$ clearly "declaring their interest". When the other two equations with $k=0,1$ are handled similarly it appears that the points of $\omega_{2}(\alpha)$ satisfy

$$
\frac{x_{0} x_{4}-4 x_{1} x_{3}+3 x_{2}^{2}}{1}=\frac{x_{0} x_{5}-3 x_{1} x_{4}+2 x_{2} x_{3}}{-2 \alpha}=\frac{x_{1} x_{5}-4 x_{2} x_{4}+3 x_{3}^{2}}{\alpha^{2}}
$$

so that, by (3.1), $\omega_{2}(\alpha)$ lies wholly on $Q$ when $p+2 q \alpha+r \alpha^{2}=0$. The two osculating planes concide when $Q$ is a cone.

An alternative identification of these two $\omega_{2}$ uses the fact that $\omega_{2}(t)$ is spanned by (Section 2) $a, b$ on $\omega_{1}(t)$ and any third point $c$ of $\omega_{2}(t)$ not collinear with them; $\omega_{2}(t)$ is traced by $\lambda a+\mu b+v c$ as $\lambda, \mu, v$ vary. Since $\omega_{2}(t)$ meets each quadric $Q$ of $N$ in $\omega_{1}(t)$ repeated the substitution of the six members of the coordinate vector $\lambda a+\mu b+v c$ for the 
$x_{i}$ in (3.1) must either produce zero, when $\omega_{2}(t)$ would lie on $Q$, or give $v^{2}=0$. Now $\omega_{2}(\alpha)$ meets $x_{2}=x_{3}=0$, which is skew to $\omega_{1}(\alpha)$ save when $\alpha$ is 0 or $\infty$, at $\left(3,-\alpha, 0,0, \alpha^{4}\right.$, $\left.-3 \alpha^{5}\right)$ and the multiplier of $v^{2}$ after the substitution from this vector is

$$
p\left(3 \alpha^{4}\right)-q\left(-9 \alpha^{5}+3 \alpha^{5}\right)+r\left(3 \alpha^{6}\right)
$$

or

$$
3 \alpha^{4}\left(p+2 q \alpha+r \alpha^{2}\right)
$$

Thus $\omega_{2}(\alpha)$ lies on that pencil of $Q$ for which $p+2 q \alpha+r \alpha^{2}=0$ while each $Q$ contains two $\omega_{2}(\alpha)$ which, as above, coincide when $Q$ is a cone.

\section{The generalisation}

7. There is a strictly analogous interpretation of the quadratic covariant $(a b)^{2 m} a_{x} b_{x}$ of a binary form of order $2 m+1$, as well as of the discriminant $\Delta$ of this quadratic. As the geometry for $m=2$ has been described at length it will suffice to state the facts for higher values of $m$ without elaboration.

All the $\omega_{m-1}$ of a rational normal curve $C$ in $[2 m+1]$ lie on the quadrics $Q$ of a net $N\left[1\right.$, p. 142]. These $\omega_{m-1}$ generate [9, p. 201] an $\Omega_{m}^{m(m+2)}$ lying on the $\Omega_{m+1}^{(m+1)^{2}}$ generated by the $\omega_{m}$, and $\omega_{m}(t)$ is the tangent $[m]$ of $\Omega_{m}^{m(m+2)}$ at every point of $\omega_{m-1}(t)$. Each $\omega_{m}$ is on a pencil of $Q$ and meets those $Q$ on which it does not lie in $\omega_{m-1}$ repeated; each $Q$ contains two $\omega_{m}$ which coincide when $Q$ is a cone. The cones of $N$ are point-cones with vertices on $C$, and their envelope is a quartic primal $K$. If $P$ lies on $K$ it maps a binary $(2 m+1) \cdot i c$ for which the invariant $\Delta$ is zero. $K$ contains $\Omega_{m+1}^{(m+1)^{2}}$.

All elements of the symmetric matrix for $Q$ are zero save those in the secondary diagonal and its two contiguous parallels. In these parallels the binomial coefficients $\left(\begin{array}{c}2 m \\ k\end{array}\right)$ appear with alternating signs; the coefficients in the secondary diagonal itself give zero when added to the two contiguous ones in the same row or column. For $m=3$ the matrix is

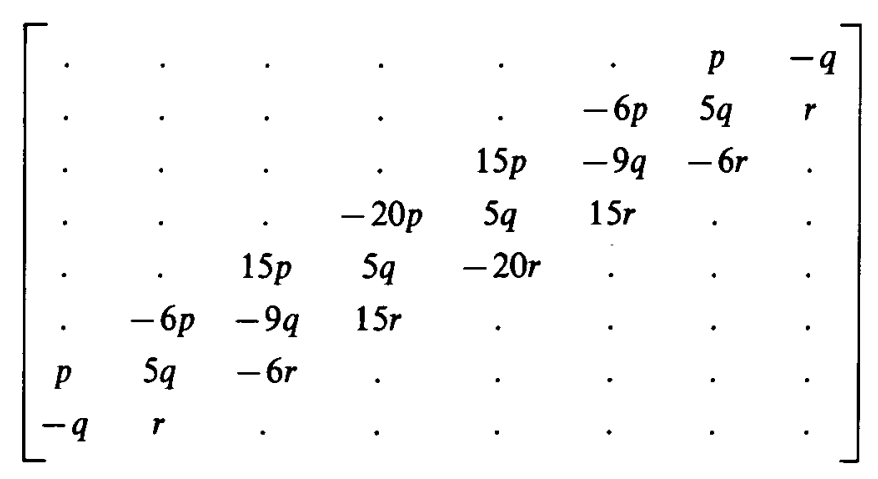

with determinant a multiple of $\left(q^{2}-r p\right)^{4}$ and, should $q^{2}=r p$, rank 7 . So the quartic 
invariant of $a_{x}^{7}$ appears on replacing $x_{i}$ by $a_{i}$ in

$$
\begin{aligned}
& \left(x_{0} x_{7}-5 x_{1} x_{6}+9 x_{2} x_{5}-5 x_{3} x_{4}\right)^{2} \\
& =4\left(x_{0} x_{6}-6 x_{1} x_{5}+15 x_{2} x_{4}-10 x_{3}^{2}\right)\left(x_{1} x_{7}-6 x_{2} x_{6}+15 x_{3} x_{5}-10 x_{4}^{2}\right)
\end{aligned}
$$

and so reproducing the expression given by Cayley $[4$, p. 316]. Of course all the coefficients occurring here are patent in

$$
(a b)^{6} a_{x} b_{x} \equiv\left(a_{1} b_{2}-a_{2} b_{1}\right)^{6}\left(a_{1} x+a_{2} y\right)\left(b_{1} x+b_{2} y\right)
$$

but the geometry surely merits being recognised.

\section{REFERENCES}

1. H. F. BAKER, Symbolical algebra and the quadrics containing a rational curve, Proc. Edin. Math. Soc. (1) 44 (1926), 131-143.

2. L. BRusottr, Sulla curva razionale normale dello spazio a quattro dimensioni, Annali di matematica (3) 9 (1904), 311-352.

3. A. Cayley, A second memoir on quantics, Phil. Trans. Roy. Soc. 146 (1856), 101-126. Collected Papers II, 250-275.

4. A. Cayley, A third memoir on quantics, ibid. 627-647. Collected Papers II, 310-335.

5. J. H. Grace and A. Young, The algebra of invariants (Cambridge University Press, 1903).

6. G. Marletta, Sulle curve razionali del quinto ordine, Palermo Rendiconti 19 (1905), 94-119.

7. G. SALMON, Lessons introductory to the modern higher algebra (Dublin; Hodges, Foster \& Co., 1876).

8. J. A. Todd, The geometry of the binary quintic form, Proc. Cambridge Phil. Soc. 40 (1944).

9. G. Veronese, Behandlung der projekțivischen Verhältnisse der Raüme von vershiedenen Dimensionen durch das Prinzip des Projizierens und Schneidens, Math. Annalen 19 (1882), 161234.

Montague House

67 Mill Hill

Musselburgh, EH21 7RL

SCOTLAND 